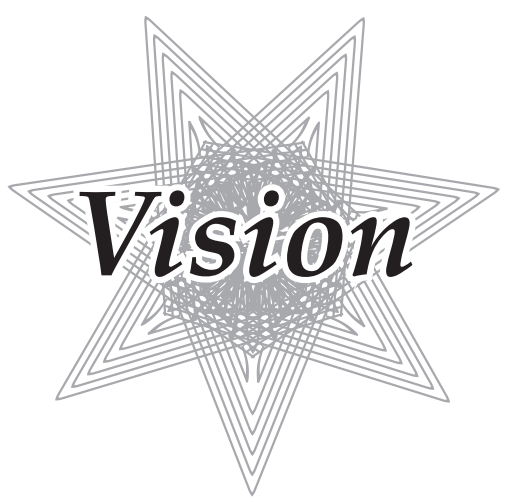

\title{
More Capacitors for Energy-Harvesting
}

\author{
Masayuki MORITA
}

The invention of Electric Double Layer Capacitor (EDLC) by General Electric, USA, in 1957 opened the door to a new world of advanced electronics. Also the commercialization of EDLCs by Japanese companies, Matsushita (Panasonic) and NEC, in the 1970s promoted practical developments in electronic device applications. Since then, EDLCs have taken active parts in wide application areas, such as memory-backup, actuators, and auxiliary power source utilities. Now, we have a variety of Electrochemical Capacitors (ECs) including EDLC, pseudo-capacitors, hybrid capacitors, etc., and expect that those devices will take more important positions in wider application areas, especially in so-called "Energy-Harvesting" systems.

The EC system has such merits as higher power density and longer cycle life than conventional battery systems, whereas lower energy density than that of batteries has often been pointed out as the demerit. Thus, many attempts have so far been proposed to improve the energy density of the capacitor device under keeping high power capability. A hybrid capacitor system, also called "Lithium-ion Capacitor (LIC)", consisting of a lithium-ion battery (LIB) negative electrode and an EDLC positive electrode, would be a highlighted example of the advanced capacitors having improved energy density.

In The Electrochemical Society of Japan (ECSJ), the Committee of Capacitor Technology was founded in 1993, and has played a prominent part in the development of science and technology of capacitors. The Committee has sponsored academic symposia on EC and conventional capacitors, which were periodically held with and without cosponsorship of Annual Meetings of ECSJ. The Committee has also promoted the International Conference on Advanced Capacitors (ICAC). The 4th Conference (ICAC2013) was held at Osaka in this May, in which about 130 papers were presented and more than 250 attendees participated from all over the world. We have also an important international symposium on advanced capacitors, ISEECap, which has been held in Europe in every two years. In the recent annual meetings of ECS (The Electrochemical Society) and ISE (The International Society of Electrochemistry), symposia dedicated to ECs have been organized in each energy conversion division. Thus, we have so many opportunities to meet progresses in capacitor science and technologies. Main topics presented in those international conferences and symposia have been on advanced materials for EC systems. For example, preparation and characterization of transition metal oxides other than conventional ruthenium oxide have attracted much attention in the last decade for applying to pseudo-capacitor electrodes. Also a variety of structure-controlled carbon materials with high specific surface area have been developed using specific raw materials and/or well-designed processes, and examined as the advanced EDLC electrodes. Carbon materials that can meet with high voltage operation in nonaqueous media have also attracted interest from the viewpoint of the enhancement in energy density.

It is well recognized that the innovation in material science is essential to the significant improvements in the device performances of the capacitor system. In this context, collaboration among the researchers on material science, device makers, and users will lead to establishing the novel system with advanced capacitor performances. In general, potential users who have looked for efficient power sources had still less knowledge on the characteristics of advanced EC system. Thus, we need "Matchmakers" for the capacitor utilization, who should have specific ability both in researching the market and in managing the capacitor technology. They can promote the efficient collaboration work among those who concern the utilization of EC in newly developed application area, especially for "Energy-Harvesting" utilities. One of the tasks of the Capacitor Committee might be to cultivate and support such "Matchmakers" for capacitor technology.

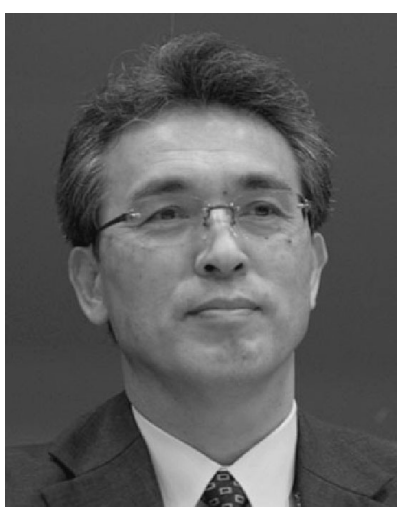

Masayuki MORITA

Professor,

Graduate School of Science and Engineering, Yamaguchi University 